\title{
Histological grading of primary bone tumors
}

\author{
Edward F. McCarthy
}

Published online: 23 August 2009

(C) ISS 2009

In 1897, David Paul Hansemann, a colleague of Rudolph Virchow, described microscopic features characteristic of malignant neoplasms that included variations in nuclear size, shape, and chromaticity and the presence of many and abnormal mitotic figures. He called these features anaplasia. He illustrated and expanded his description of anaplasia in his textbook of 1897, The Microscopic Diagnosis of Malignant Tumors [3]. This concept was reiterated by James Ewing in the first edition of his book Neoplastic Diseases in 1919 [2]. The following year, Albert Borders of the Mayo clinic, postulated that the severity of anaplasia in squamous cell carcinoma of the lip was related to the prognosis [1]. Carcinomas with little anaplasia did better than those with severe anaplasia. Over the years, he extended this idea to other epithelial neoplasms. This was the origin of a process known as histological grading - using microscopic features to predict the aggressiveness of a neoplasm. This concept of grading is now used in the diagnosis of virtually all human and most animal cancers.

There are many grading systems, but almost all include common features: the degree of nuclear atypia, the number of mitotic figures, the presence of abnormal mitotic figures, the degree of cellularity, the presence or absence of encapsulation, and the amount of tumor necrosis. Most systems have three grades, from grade 1 (low-grade) to grade 3 (high-grade). However, some systems have four grades. Histological grade is distinguished from the concept of "differentiation," which evaluates how closely a neoplasm resembles normal tissue. Poorly differentiated neoplasms have little resemblance to normal tissue, whereas

\footnotetext{
E. F. McCarthy $(\bowtie)$

Departments of Pathology and Orthopedic Surgery,

The Johns Hopkins Hospital,

401 North Broadway,

Baltimore, MD 21231, USA

e-mail: mccarthy@jhmi.edu
}

well-differentiated neoplasms may sometimes be difficult to distinguish from non-cancerous tissue. Occasionally, the differentiation of a tumor is included in the grading system.

Two problems arise in the management of malignant neoplasms: local recurrence after resection and metastasis. Local recurrence is primarily a function of the adequacy of the surgical resection. Wide, tumor-free margins usually prevent local recurrence. The other problem, the likelihood of metastasis, can usually be predicted by the histological grade. Therefore, grading a neoplasm aids in determining prognosis and in deciding whether systemic chemotherapy to prevent metastasis is required. High-grade (grade 3) neoplasms are more likely to metastasize, and systemic chemotherapy may be useful to prevent this dissemination. Low-grade malignancies have a low incidence of metastasis and chemotherapy may not be appropriate.

The concept of grading worked its way slowly into bone tumor pathology. Henry Jaffe, in his seminal book of 1958, Tumors and Tumorous Conditions of Bones and Joints, used the concept of well-differentiated and poorly differentiated, but he did not mention histological grading except in cases of giant cell tumor of bone, an aggressive benign tumor [5]. For many years, grading was a common exercise in the diagnosis of giant cell tumor of bone to predict how aggressive the lesion might be. However, grading of giant cell tumor of bone is no longer used; giant cell tumors are felt to be either conventional or malignant. Malignant giant cell tumors are high-grade sarcomas with a high rate of metastasis. Curiously, conventional benign giant cell tumor, although regarded as "benign," may metastasize, but histological grade is not considered indicative of metastatic potential. Factors such as the number of local recurrences, an axial location, and soft tissue spread are better predictors of "benign metastasis."

Nowadays, only malignant bone tumors are graded. The histological grade, usually 1 through 3 , is useful to 
determine the prognosis and in many cases to decide whether adjuvant therapy is necessary. Grading is done on osteosarcomas, chondrosarcomas, fibrosarcomas, and vascular malignancies. Some bone tumors are not graded, such as Ewing's sarcoma, which are all considered highgrade [8]. Also, chordomas and adamantinomas are not graded because cytological features do not predict behavior in these neoplasms [4].

Chondrosarcomas are graded for prognosis only. Chondrosarcomas may be grouped into grades 1 through 3. Most chondrosarcomas are grades 1 and 2. Only $10 \%$ of chondrosarcomas are grade 3. Chondrosarcomas are not sensitive to radiotherapy or chemotherapy. Therefore, the histological grade is used for prognosis only, and the practice of grading is accurately predictive. Metastases occur in over $50 \%$ of grade 3 lesions, whereas only 10 to $15 \%$ of grade 1 and grade 2 chondrosarcomas metastasize [7].

Histological grading is important in the management of osteosarcomas, although the grade is often implied when the subtype of osteosarcoma is diagnosed. For example, most osteosarcomas are conventional osteoblastic osteosarcomas. Conventional osteosarcomas are all grade 3 (high-grade), and they will all require chemotherapy. This is also true of telangiectatic osteosarcoma. At the other end of the spectrum, parosteal osteosarcoma and well-differentiated intraosseous osteosarcoma are grade 1 neoplasms and have a low incidence of metastasis. These fibroblastic lesions do not require neoadjuvant or post-operative chemotherapy. A third variant, periosteal osteosarcoma is an intermediate-grade (grade 2) osteosarcoma and requires preoperative chemotherapy [6].

Fibrosarcomas are also graded, and the histological grade will determine the need for chemotherapy. Low-grade (grade 1) fibrosarcomas are uncommon and generally do not require chemotherapy. High-grade fibrosarcomas, do require neoadjuvant chemotherapy. There has been a trend among soft tissue pathologists to replace the diagnostic category of malignant fibrous histiocytoma with high-grade fibrosarcomas. However, when the diagnosis of malignant fibrous histiocytoma is rendered, a grade should also be included. Almost all malignant fibrous histiocytomas are grade 3 malignancies, and grading will allow the very rare benign fibrous histiocytoma to be excluded. In addition to fibrosarcomas, another malignant spindle cell neoplasm, the rare primary leiomyosarcoma of bone, should also be graded.

Finally, primary malignant vascular neoplasms of bone should be graded. Of these rare neoplasms, the most commonly encountered are epithelioid hemangioendotheliomas. These lesions are grade 1 to grade 2 and may require adjunctive chemotherapy. The extremely rare highgrade angiosarcoma of bone has a high potential for metastasis and should be treated with chemotherapy.

Accurately ascribing a grade to a neoplasm is problematic when small needle biopsy fragments are analyzed. High- grade cellular features generally correctly characterize a neoplasm as aggressive. However, because neoplasms are heterogeneous, findings of low-grade features may not reflect that a possible adjacent high-grade area has not been sampled, and therefore an accurate prediction of cellular growth cannot be determined. Therefore, to have the greatest chance of accuracy, CT-guided core biopsies should sample at least three different areas.

Two special problems arise in grading bone neoplasms. First, because in low-grade lesions the features of anaplasia may only be minimal, distinguishing a low-grade neoplasm from a benign bone lesion may be difficult . For example, a well-differentiated (grade 1) fibroblastic intraosseous osteosarcoma may be misdiagnosed as fibrous dysplasia. Also, a low-grade chondrosarcoma may be confused with an enchondroma. Second, some benign bone tumors, such as chondromyoid fibroma, show significant reactive cellular atypia and may be misdiagnosed as a chondrosarcoma. This misdiagnosis can be avoided if the pathologist notices that other features of anaplasia, such as mitotic figures, are not present. Both of these special problems can be avoided if the radiographic presentation is carefully studied. Lowgrade histological features in a lesion with a very aggressive radiographic pattern should alert the pathologist that perhaps the lesion has not been adequately sampled. Conversely, if a lesion appears to have high-grade histology and the radiograph suggests an indolent lesion, then the pathologist might consider that the atypia is reactive.

Histological grading is a task performed by pathologists. The histological grade in conjunction with radiographic features and the adequacy of surgical excision interplay to determine therapy and outcome. This interplay highlights the necessity of the team approach in the management of bone tumors.

\section{References}

1. Broders AC. Squamous-cell epithelioma of the lip: a study of five hundred and thirty-seven cases. J Am Med Assoc. 1920;74 (10):656-64.

2. Ewing J. Neoplastic diseases: a text-book on tumors. Philadelphia: Saunders; 1919.

3. Hansemann D. The microscopic diagnosis of malignant tumors. Berlin: Hirschwald; 1897.

4. Inwards CY, Unni KK. Classification and grading of bone sarcomas. Hematol Oncol Clin North Am. 1995;9(3):545-569.

5. Jaffe HL. Tumors and tumorous conditions of the bones and joints. Philadelphia: Lea \& Febiger; 1958.

6. Klein MJ, Siegal GP. Osteosarcoma: anatomic and histologic variants. Am J Clin Pathol. 2006;125(4):555-81.

7. Rizzo M, Ghert MA, Harrelson JM, Scully SP. Chondrosarcoma of bone: analysis of 108 cases and evaluation for predictors of outcome. Clin Orthop Relat Res. 2001;391:224-33.

8. Unni KK, Dahlin DC. Grading of bone tumors. Semin Diagn Pathol. 1984;1(3):165-72. 